\title{
Prediction of Particle Distribution in Isotropic Turbulence by Large-Eddy Simulation
}

\author{
Y. Yang*, G. W. He, G. D. Jin \\ LNM, Institute of Mechanics, Chincse Academy of Sciences, Beijing 100080, China \\ Email: yangyue@lnm.imech.ac.cn
}

\begin{abstract}
The recent application of large-eddy simulation (LES) to particle-laden turbulence requires that the LES with a subgrid scale (SGS) model could accurately predict particle distributions. Usually, a SGS particle model is used to recover the small-scale structures of velocity fields. In this study, we propose a rescaling technique to recover the effects of small-scale motions on the preferential concentration of inertial particles. The technique is used to simulate particle distribution in isotropic turbulence by LES and produce consistent results with direct numerical simulation (DNS).
\end{abstract}

Key words: particle distribution, particle-laden turbulence, large-cddy simulation, subgrid scale model.

\section{INTRODUCTION}

The preferential concentration of inertial particles is important in turbulent particle-laden flows. For example, the local accumulation of droplets in warm cloud would enhance the droplet growth by collision and coalescence [1]. Recently, large-eddy simulation (LES) has been applied to simulate particle-laden turbulence [2, 3]. In LES, large-scale motions are directly computed while small-scale or subgrid scale (SGS) motions are not explicitly represented but their effects on large scales are modeled. It has been known that such a SGS velocity model could ensure the correct prediction of LES on velocity energy spectra at large scales. However, such kind of SGS velocity models may not correctly predict the particle distributions. The reason is as follows: the particles in turbulent flows tend to accumulate in the low-vorticity regions due to the inertial bias and thus exhibit a preferential concentration $[4,5]$. The preferential concentration is very much dependent on SGS motions at intermediate Stokes numbers. The effect of SGS motions on particle distribution cannot be represented by the SGS velocity models [6] and instead, SGS particle models are being developed for this purpose.

Recently, Shotorban and Mashayek [7] used the deconvolution approach to compute the preferential concentration in turbulent channel flows. The deconvolution approach can partially recover the unsolved velocity field. Fade and Simonin [8] pointed out that the effects of SGS motions on the preferential concentration lead to three kinds of behavior depending on a subgrid Stokes number, which implies that there exist discrepancies in preferential concentration results between the direct numerical simulation (DNS) and LES.

In this study, we will use a LES with a spectral eddy-viscosity SGS model to predict preferential concentrations of inertial particles in isotropic turbulence. And the results obtained from LES will be compared with those from DNS. Based on the comparison, we propose to rescale the response time scale of the particles to ensure the correct prediction on preferential concentrations. The technique is verified via numerical simulations.

\section{SIMULATION OVERVIEW}

The stationary and homogeneous isotropic turbulence is simulated by DNS with the grid size $128^{3}$ and by LES with the grid size $32^{3}$ on a cubic of side $2 \pi$. A standard pseudo-spectral method is used. 
In LES, we use the Chollet-Lesieur standard form for the spectral eddy-viscosity model. The details of these numerical methods are given in Ref. 9. Figure 1 plots the resulting energy spectra for DNS $128^{3}$ and LES $32^{3}$. Table 1 lists Eulerian statistics of the DNS and LES flow fields used in this study.

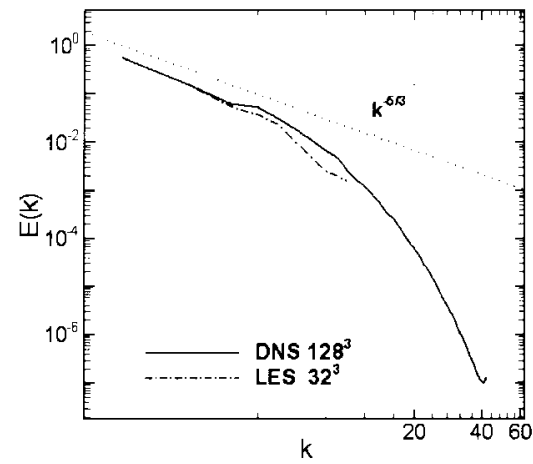

Table 1: Summary of data of the flow fields

\begin{tabular}{lcc}
\hline & DNS $128^{3}$ & LES $32^{3}$ \\
\hline Taylor Reynolds number $\operatorname{Re}_{\lambda}$ & 60 & - \\
Rms fluctuating velocity $u^{\prime}$ & 0.788 & 0.753 \\
Dissipation rate $\varepsilon$ & 0.152 & - \\
Kolmogorov length scale $\eta$ & 0.051 & - \\
Kolmogorov time scale $\tau_{\eta}$ & 0.257 & - \\
Eulerian integral time scale $L_{E}$ & 1.74 & 1.85 \\
Eddy turnover time $T_{e}$ & 2.21 & 2.46 \\
\hline
\end{tabular}

Figure 1: Energy spectra

A three-dimensional sixth-order Lagrangian interpolation scheme is used to compute fluid velocity at the location of a particle. The trajectory of an individual particle is obtained by the numerical integration of the particle kinematic equation $\mathrm{d} X / \mathrm{d} t=V$ for particle's position and the simplified BBO equation $\mathrm{d} V / \mathrm{d} t=(\boldsymbol{u}-\boldsymbol{V}) / \tau_{p}$ for particle's velocity, where $\boldsymbol{X}$ and $\boldsymbol{V}$ are particle position and velocity respectively, $\boldsymbol{u}$ is the fluid velocity at the particle position and $\tau_{p}$ is the particle's aerodynamic response time. In this study, the diameter of inertial particle is assumed to be small in comparison with the Kolmogorov length scale of turbulence and dilute enough so that it does not modify the turbulent flow field. The simulation of this type of inertial particle is very useful in turbulent particle-laden flows, especially for the particle motion in atmospheric turbulence.

\section{PREFERENTIAL CONCENTRATION IN LES}

Particle concentration $C$ is defined here as the number of particles found inside a small cube and its side length equals the grid spacing of LES $32^{3}$. The probability distribution function (PDF) of the concentration $P_{C}(C, t)$ is the probability of finding that the concentration at the small cube equals to a particular value $C$. At the initial time, the distribution of particles is uniform, so $P_{C}$ approximately satisfies the Poisson distribution

$P_{C}^{u}(C, t=0)=\langle C\rangle^{C} e^{-\langle C\rangle} / C$ !

where the average concentration in the computational domain $\langle C\rangle$ was chosen as unity. The probability for the high concentration should increase with time during the transient period, since inertial particles tend to accumulate in low vorticity and high strain regions due to the inertial bias $[4,5]$. The local accumulation $D_{c}$ can be measured via the square derivation of the PDFs of particle distributions.

$D_{C}(t)=\sum_{C=0}^{N_{p}}\left(P_{C}(C, t)-P_{C}^{u}(C, t=0)\right)^{2}$

Figure 2 shows the temporal evolutions of $D_{C}$ in DNS with three typical Stokes numbers. Here, the Stokes number is defined as $S t=\tau_{p} / \tau_{\eta}$. At $t=0$, the uniform distribution of particles implies $D_{C}=0$. The increasing of $D_{C}$ during the transient period indicates the local accumulation of particles increases. Finally, the concentration field approaches a statistical stationary state after about two eddy turnover times.

We compared the temporal evolution of $D_{c}$ obtained in DNS and LES respectively with different Stokes numbers in Figure 2. When $S t$ is less than about 1.2, LES underpredicts the preferential concentration, but when $S t$ is greater than 1.2, LES overpredicts the quantity. To study the longtime trends of the preferential concentration, $\left\langle D_{C}\right\rangle$, averaged over the stationary stage, is plotted against the Stokes number in Figure 3. In DNS, $\left\langle D_{C}\right\rangle$ reaches a maximum around $S t \approx 1.0$ as former results [5], while in LES, the curve has a deviation from that of DNS.

Maxey's asymptotic analysis [4] for small inertial particles showed that the local particle concentration would evolve as 
$C(\boldsymbol{x}, t)=C_{0} \exp \left\{\tau_{p} \int_{0}^{t} Q\left(\boldsymbol{X}, t^{\prime}\right) \mathrm{d} t^{\prime}\right\}$

where $Q=\frac{\partial u_{i}}{\partial x_{j}} \frac{\partial u_{j}}{\partial x_{i}}$. The integration in the right hand side of Eq. (3) is evaluated following a particle's Lagrangian trajectory. Through the dimension analysis

$\int_{0}^{t} Q\left(X, t^{\prime}\right) \mathrm{d} t^{\prime} \sim[T]^{-1}$

the local particle concentration is related to a characteristic time scale of the flow field. We assume that this time scale is $\tau_{\eta}$. Thus, the local concentration should be a function of the Stokes number as

$C(\boldsymbol{x}, t)=C_{0} \exp \{f(S t, t)\}$

which implies that the Stokes number is the most critical parameter to particle preferential concentration. In LES, the dissipation rate $\varepsilon_{L E S}$ which can be resolved is underpredicted, which leads to an overprediction of the smallest time scale $\tau_{\eta}^{L E S}$ which can be resolved in the LES. Here, $\tau_{\eta}^{L E S}$ is calculated as

$\tau_{\eta}^{L E S}=\sqrt{\nu / \epsilon_{L E S}}=\sqrt{1 / 2 \int_{0}^{k_{c}} k^{2} E(k) \mathrm{dk}}$

where $E(k)$ is the energy spectrum and $k_{\mathrm{c}}$ the cutoff wavenumber in LES. Therefore, compared with the same inertial particles in DNS, their Stokes numbers in LES are underpredicted, which causes the discrepancies in the preferential concentration results between DNS and LES as shown in Figure 2 and Figure 3.

\section{A RESCALING TECHNIQUE FOR PARTICLE DISTRIBUTION IN LES}

There are two approaches to recover the effects of SGS motions on particle distributions. The first approach is to reconstruct the velocity fields $[3,7]$. It requires not only spatial statistics but also spatial structures in LES velocity fields. That is a very challenging task. Another approach is to rescaling the response time scale of particles in LES, which will be described in details as follows.

The basic idea in the rescaling approach is to dynamically increase particle's response time $\tau_{p}$ in LES. The increase corresponds to the overprediction of the Kolmogorov time scale $\tau_{\eta}$ in LES. It ensures the Stokes number in LES, the most critical parameter to particle-vortex interaction as shown in Eq. (5), is similar as that in DNS. The modeled response time $\tau_{p}^{+}$is calculated as

$\tau_{p}^{+}=\frac{\tau_{\eta}^{L E S}}{\tau_{\eta}^{D N S}} \tau_{p}^{\approx} \frac{\tau_{\eta}^{L E S}}{\tau_{\eta}^{+}} \tau_{p}$

To obtain a good approximation $\tau_{\eta}^{+}$of the Kolmogorov time scale in DNS using the LES data, the SGS dissipation rate $\varepsilon_{S G S}$ should be included as

$\tau_{\eta}^{+}=\sqrt{\nu /\left(\varepsilon_{L E S}+\varepsilon_{S G S}\right)}=\sqrt{\nu / 2 \int_{0}^{k_{c}}\left[\nu+\nu_{t}\left(k \mid k_{c}\right)\right] k^{2} E(k) \mathrm{d} k}$

Here, $\nu_{t}$ is the eddy-viscosity in the spectral eddy-viscosity SGS model. Then the new dynamical equation for particle is $\mathrm{d} V / \mathrm{d} t=(\boldsymbol{u}-V) / \tau_{p}^{+}$. It turns out that the Stokes number in the LES with our proposed model is $\tau_{p}^{+} / \tau_{\eta}^{L E S}$, which is similar as that in DNS.

As shown in Figure 2, LES with the proposed model can predict the time evolution of $D_{C}(t)$ more accurately than those obtained from the ordinary LES. Moreover, in Figure 3, the proposed model can effectively correct the deviation in the LES curve from that of DNS via rescaling the response time in Eq. (7). Thus, LES with the proposed model can obtain more accurate preferential concentration in the stationary stage over a wide range of Stokes numbers. 


\section{CONCLUSIONS}

One of the main challenges to LES of particle-laden turbulence is to predict the smallest time scale of the flow fields, which determines the Stokes number in practice. Our strategy is to rescale the response time scale of particle in LES to ensure the Stokes number is similar as that in DNS, instead of using a SGS particle model. The latter does not to reproduce small-scale structures of velocity fields and thus is hard to improve the prediction of the preferential concentrations. The rescaling strategy is verified to make a good approximation to the Kolmogorov time scale in DNS and well predict the preferential concentrations.

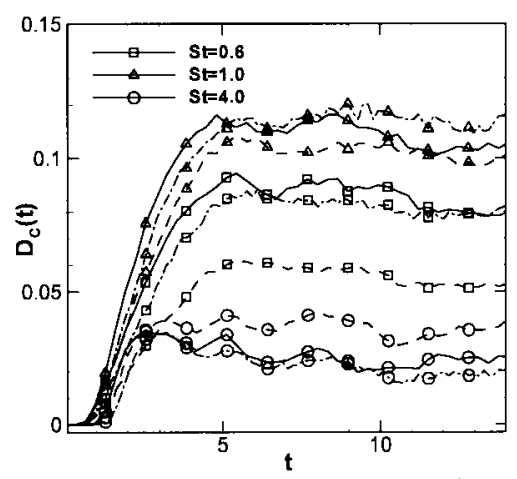

Figure 2: Temporal evolutions of the global measures of local accumulation: — DNS $128^{3}-\operatorname{LES} 32^{3}-\cdot-\operatorname{LES} 32^{3}$ with proposed model. The symbols indicate three typical Stokes numbers.

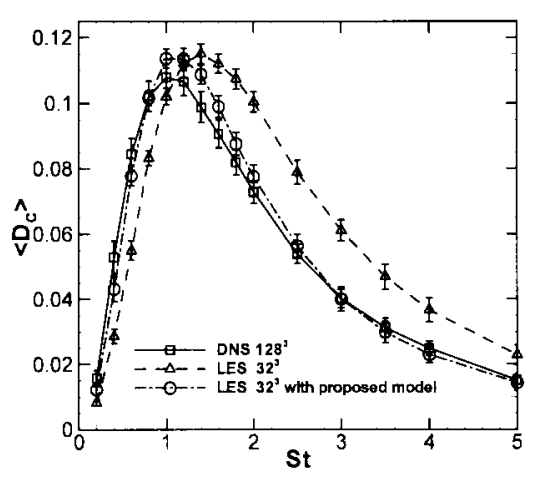

Figure 3: Time-averaged global measures of local particle accumulation as a function of the particle Stokes number. The error bars indicate standard deviations.

\section{ACKNOWLEDGEMENT}

This work was supported by Chinese Academy of Sciences under the innovative project "Multi-scale modelling and simulation in complex system" (KJCX-SW-L08) and National Natural Science Foundation of China under the Project Nos. 10325211 and 10628206.

\section{REFERENCES}

1. Shaw RA. Particle-turbulence interactions in atmospheric clouds. Annu. Rev. Fluid Mech., 2003;35:183-227

2. Loth E. Numerical approaches for motion of dispersed particles, droplets and bubbles. Prog. Energy. Combust. Sci., 2000;26:161-223

3. Mashayek F, Pandya RVR. Analytical description of particle/droplet-laden turbulent flows. Prog. Energy. Combust. Sci., 2003;29:329-378

4. Maxey MR. The gravitational settling of aerosol particles in homogeneous turbulence and random flow fields. J. Fluid Mech., 1987;174:441-465

5. Wang LP, Maxey MR, Settling velocity and concentration distribution of inertial particles in homogeneous isotropic turbulence. J. Fluid Mech., 1993;256:27-68

6. Yang Y, He GW, Wang LP. Effects of subgrid scale modeling on Lagrangian statistics in large-eddy simulation. Phys. Fluids, 2007

7. Shotorban B, Mashayek F. Modeling subgrid-scale effects on particles by approximate deconvolution. Phys. Fluids., 2005;17:081701

8. Fede P, Simonin O. Numerical study of the subgrid fluid turbulence effects on the statistics of heavy colliding particles. Phys. Fluids, 2006;18:045103

9. He GW, Rubinstein R, Wang LP, Effects of subgrid-scale modeling on time correlations in large eddy simulation. Phys. Fluids, 2002;14:2186-2193 